\title{
La composante visuelle de l'écriture scientifique : vers une analyse des formes
}

Tracing forms in science writing: a study of non-verbal elements

\section{Gwen Le Cor}

\section{(2) OpenEdition}

1 Journals

Édition électronique

URL : http://journals.openedition.org/asp/4721

DOI : 10.4000/asp.4721

ISSN : 2108-6354

Éditeur

Groupe d'étude et de recherche en anglais de spécialité

\section{Édition imprimée}

Date de publication : 23 octobre 2015

Pagination : 87-102

ISSN : 1246-8185

\section{Référence électronique}

Gwen Le Cor, "La composante visuelle de l'écriture scientifique : vers une analyse des formes », $A S p$ [En ligne], 68 | 2015, mis en ligne le 01 novembre 2016, consulté le 04 novembre 2020. URL : http:// journals.openedition.org/asp/4721; DOI : https://doi.org/10.4000/asp.4721

Ce document a été généré automatiquement le 4 novembre 2020.

Tous droits réservés 


\title{
La composante visuelle de l'écriture scientifique : vers une analyse des formes
}

Tracing forms in science writing: a study of non-verbal elements

\author{
Gwen Le Cor
}

1 L'écriture scientifique comporte une composante visuelle spécifique, qui se décline sous forme d'équations, de graphes, de schémas, de représentations de liens chimiques, ou de diagrammes. Ces aspects «multi-sémiotiques » ou « multimédia » (« semiotic hybrids » Lemke $2005: 87$; « combinatorial semiotic principle ${ }^{1}$ » Lemke $\left.2005: 92\right)$ ont souvent été convoqués par les chercheurs pour souligner l'importance des modes de transmission non verbaux de la connaissance en science. Dès 1997, Claude Sionis présente les éléments non verbaux comme des vecteurs d'information, et leur confère ainsi un statut distinct de celui de simple illustration picturale :

[The researchers'] verbal discourse was created and organised to develop and comment on NVM [non-verbal material] and not the other way around. NVM were definitely not used to "illustrate" a predominantly verbal type of communication; they were the very subject and the main mode of communication. (1997 : §2)

Dans un article qui s'attache à définir le genre des présentations orales lors de conférences scientifiques, Elizabeth Rowley-Jolivet retient le rôle prépondérant de la «communication visuelle» dans les exposés scientifiques : «A striking feature of the scientific conference paper is the importance of the visual channel of communication " (2002: 20). Ces deux arguments soulignent l'impact communicationnel des visuels projetés ; pourtant, l'hybridité sémiotique de l'écriture scientifique nous entraîne audelà d'une rhétorique communicationnelle. Cet article se propose donc de déplacer légèrement le curseur pour envisager ce qui fait «image » ou "forme » en science. Il s'agira d'examiner comment les formes font sens en science, voire comment elles font science, et de montrer que l'image ou la forme sont des modes fondamentaux de la pensée scientifique qui façonnent les traces écrites que nous livrent les scientifiques. 
3 Précisons auparavant ceci : dans l'éditorial du numéro 66 de la revue ASp, Anthony Saber désigne l'anglais de spécialité comme une « discipline d'interface » et s'interroge en retour sur le risque d'éclatement que les intersections comportent : « Ne faudrait-il pas [que l'anglais de spécialité] trouve les voies et moyens pour se transformer en discipline autonome, dotée de cadres conceptuels qui lui soient propres et qui permettraient de penser de manière intégrée l'intégralité des phénomènes dont il s'empare? » (\$11). En gardant ceci à l'esprit, il importe d'essayer de penser "le » discours scientifique sans forcément se limiter à un champ disciplinaire particulier ${ }^{2}$. Par discours scientifique il faut entendre ici la définition qu'en donne Michel Petit à savoir «l'ensemble des productions textuelles relevant du domaine des sciences, [...] et ressortissant ipso facto à un 'style scientifique' » (1997: § 2). Mon approche vise à dégager un trait distinctif commun à tout discours scientifique et atténue de fait les variations propres à chaque domaine. C'est en gommant les différences entre domaines que nous pouvons aller « vers une analyse des formes » comme l'indique le sous-titre de l'article.

4 Après un bref panorama des études existantes, je montre les limites des approches linguistiques centrées sur les composantes verbales du discours scientifique et m'attache à la pensée scientifique comme pensée des formes. Ces analyses m'amènent à reposer différemment le problème des outils théoriques dont disposent les anglicistes pour analyser le discours scientifique, et à proposer d'intégrer les outils plus littéraires ou philosophiques que sont ceux de l'analyse de l'image.

\section{Les limites d'une approche linguistico-discursive (textuelle)}

5 Dans une étude qui recense vingt années de recherches en anglais scientifique publiées dans la revue ASp (de 1993 à 2012), Hélène Laffont et Jean-Louis Trouillon notent une prépondérance des explorations didactiques ainsi que des analyses qui relèvent de la linguistique au sens large (2013: § 7). Un premier tableau classe les études linguistiques selon des approches «discursive, grammaticale, phonologique, terminologique et diachronique » et un deuxième tableau détaille ce que les auteurs rassemblent sous la rubrique "analyse de discours", à savoir, des études relevant du genre, de la stylistique, de la cohésion, de la métaphore, de la sémiotique, ou encore celles s'intéressant à la structure textuelle ou à l'abstract. Partant d'une visée plus spéculative, Michel Petit envisage les pistes possibles pour analyser le discours scientifique du point de vue de la linguistique, et explique que « le discours scientifique peut constituer un objet d'étude pour l'analyse de discours, la grammaire de texte, l'analyse de genre, la traductologie, la pragmatique, la lexicologie et la terminologie, etc. », domaines auquel il ajoute la stylistique (1997 : § 2-3) 3 .

6 Les approches linguistiques s'attachent donc majoritairement aux composantes verbales et ce à différents niveaux: celui du lexique (pour ce qui relève de la lexicologie, ou de la terminologie), celui de la phrase (phraséologie, ou analyse de syntaxe), et enfin celui du texte (structure textuelle globale, analyse de discours). Dès lors, c'est l'aspect textuel et discursif qui domine la recherche linguistique appliquée à l'anglais scientifique. Telle en serait aussi la limite pour définir un "style scientifique ", notion que j'emprunte à l'article de Michel Petit, et qui me semble essentielle. Pour autant, en transposant la notion de «stylistique littéraire » au discours scientifique, et 
en s'attachant au "fonctionnement du langage qui est celui du discours scientifique " (1997: § 3), M. Petit se concentre lui aussi avant tout sur sa composante textuelle. Or les chercheurs l'ont montré, les formes graphiques imprègnent les écrits scientifiques et en sont la nervure.

7 C'est sans doute paradoxalement l'analyse d'Elizabeth Rowley-Jolivet consacrée au genre de la communication orale scientifique lors de colloques (2002), qui rend le mieux compte de l'hybridité sémiotique des écrits scientifiques. Elle souligne l'omniprésence des éléments graphiques dans les visuels projetés au fil des présentations, mais revient aussi sur les caractéristiques "multi-sémiotiques » de l'écrit: "Semioticians, historians and sociologists of science, as well as scientists themselves, have long recognized the importance of non-linguistic elements in the construction and the communication of scientific knowledge " (Rowley-Jolivet 2002 : 22). Si elle répertorie les objets non verbaux et évoque la communication non verbale, son propos n'est pas de caractériser les écrits scientifiques, mais d'examiner les productions orales et les visuels qui leur sont associés.

8 Je voudrais opérer un léger glissement et sortir du champ d'investigation traditionnellement associé à la notion de "discours scientifique». La formulation renvoie à la linguistique et à un mode de communication orale qui délimite de fait notre manière d'appréhender les traces écrites scientifiques. Le terme « trace » évoque non seulement le tracé de l'écrit, mais désigne aussi l'empreinte spectrale d'un mode de pensée et son lieu d'incubation. Mon hypothèse est la suivante : les objets graphiques sont la trace d'une pensée des formes ${ }^{4}$, dont il me faut maintenant esquisser les contours.

\section{Une pensée des formes}

9 Un premier point d'ancrage pour tester cette hypothèse passe par la manière dont les scientifiques caractérisent le cheminement de leur pensée. Dans Paraboles et Catastrophes, le mathématicien René Thom commence par s'interroger sur ce qui « est science et ce qui ne l'est pas » :

Comme les polémiques des épistémologues en témoignent il est très difficile de

"définir» la science ou même de tracer une ligne de démarcation dénuée

d'ambiguïté entre ce qui est science et ce qui ne l'est pas. (1983:5)

Pour R. Thom, le «premier objectif » de la conceptualisation scientifique consiste «à caractériser un phénomène en tant que forme, forme 'spatiale'.» Il relie ainsi l'abstraction à sa capacité à faire forme : « comprendre signifie » pour lui « avant tout géométriser » et en réciproquement «avoir recours à la géométrie, c'est également avoir recours à » une "abstraction» (1983: 6). Cette définition mathématique et topologique sert de point de départ à une explication de la théorie des catastrophes. $\mathrm{R}$. Thom élabore ainsi une "théorie morphologique, une théorie des formes", et l'on pourrait s'interroger sur la pertinence pour les autres champs disciplinaires du raisonnement qu'il présente. Toutefois, mathématiciens, physiciens et biologistes s'accordent pour dire qu'ils s'appuient davantage sur les images et les formes plutôt que sur une argumentation discursive.

11 Dans un article consacré à la pensée gestuelle, le peintre et spécialiste de physique théorique Jacques Mandelbrojt, compare la logique scientifique à celle des artistes. Pour lui « la pensée scientifique, à l'état naissant, n'est pour nombre de scientifiques, 
pas plus une pensée verbale que ne l'est celle des artistes. » (2007 : §4) J. Mandelbrojt déploie son propos en citant le biologiste et prix Nobel Jacques Monod pour qui tous les scientifiques prennent «conscience que leur réflexion au niveau profond n'est pas verbale: c'est une expérience imaginaire, simulée à l'aide de formes, de forces, d'interactions qui ne composent qu'à peine une image au sens visuel du terme" (Monod 1970: 170 cité par Mandelrojt). Ce premier stade, non verbal est celui de l'intuition, et de la pré-conceptualisation; il précède celui des traces écrites qui nous intéressent ici. Pour autant, le lien que suggère J. Monod entre formes, interactions et forces est crucial. Le sens se construit donc d'abord comme forme, il est une force et une énergétique. Quelques exemples suffiront à nous en convaincre, à commencer par les diagrammes.

12 La méthode diagrammatique (en physique) "permet de visualiser les calculs et d'arriver plus rapidement au résultat, à la simplification voulue » que par une voie algébrique (de Saint-Ours $2004: 32$ ). À titre d'exemple, le physicien Richard Feynman ${ }^{5}$ a mis au point une technique qui porte son nom, les diagrammes de Feynman (Feynman graphs ou Feynman diagrams en anglais), utilisée en physique des particules pour décrire les modes d'interaction entre particules (voir figure 1).

Figure 1. Diagrammes de Feynman

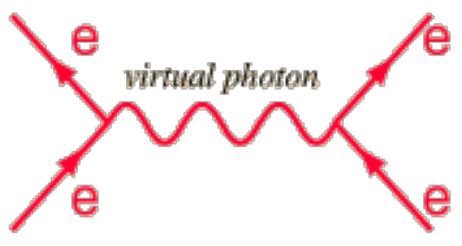

Electromagnetic

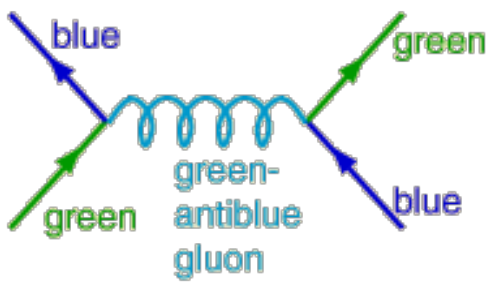

between quarks

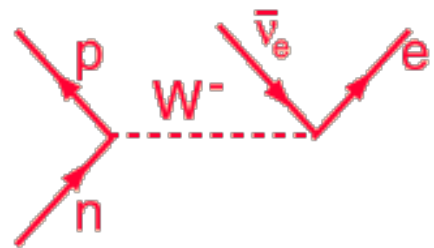

Weak

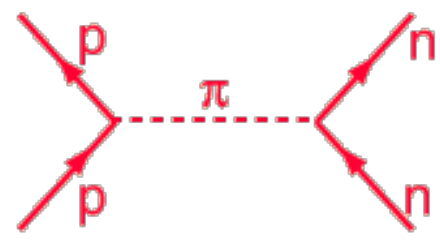

between nucleons

\section{Strong Interaction}

13 Ces diagrammes permettent de visualiser les processus de l'interaction (interactions faibles, interactions fortes ou interactions électromagnétiques), et de calculer plus facilement leur probabilité. Plus précisément, R. Feynman a mis au point ces graphes pour faciliter les calculs des « amplitudes de probabilité de transition entre deux états d'un système» (Rosenbaum 2009: § 2). Dans un article consacré au «statut des diagrammes de Feynman ", Alexis Rosenbaum explique de manière très claire comment Feynman a procédé pour « faciliter ses calculs » :

Feynman entreprit d'associer chaque terme mathématique à un graphe schématisant les événements intermédiaires ayant pu avoir lieu entre les deux états considérés. Les particules y étaient représentées par des lignes (qui pouvaient être 
dessinées de plusieurs façons en fonction du type de particule désigné) et leurs interactions par des vertex. Peu à peu, Feynman élabora ces graphes de façon plus formelle et étendit leur portée. (2009 : \$2)

Dans Quantum Field Theory, Franz Mandel et Graham Shaw ont recours à une formulation plus poétique et picturale pour décrire ce qui s'apparente à une mise en images des mathématiques : «a most useful way of picturing the mathematics » (1984: 56). Le choix des termes nous invite à nous pencher sur ce faire image mathématique, ainsi que sur la différence entre le propos de F. Mandel et G. Shaw et celui de J. Monod évoqué plus haut. J. Monod décrit le stade de l'intuition comme étant «à peine une image au sens visuel »; F. Mandel et $G$. Shaw, à l'inverse, font référence à un mode de conceptualisation par l'image, qui correspond à une seconde étape de la construction théorique. Les diagrammes n'ont pas "vocation à la fidélité figurative». Ils correspondent «à une infinité d'événements possibles» (Rosenbaum 2009: §6) qui doivent donner lieu à un calcul. Loin d'avoir un statut d'illustration, les diagrammes mettent en évidence la probabilité de rencontre entre particules élémentaires. Notons du reste la puissance de condensation de ces formes diagrammatiques. Elles présentent à la fois une sommation mathématique et un récit graphique, et n'ont besoin d'aucun texte explicatif pour être interprétées. Dans l'exemple reproduit dans la figure 1, le diagramme représentant une interaction électromagnétique (correspondant aux rayonnements visibles ou aux rayons X par exemple), "raconte l'histoire " de deux électrons: les électrons se rencontrent, échangent un photon virtuel, et repartent inchangés. L'interaction forte change la nature du neutron et du quark: le neutron devient proton, et le quark vert devient quark bleu. La «couleur» des quarks ne renvoie nullement aux couleurs telles que le langage courant les définit, il s'agit d'une analogie pour figurer une "charge " admettant trois états (là où la charge électrique n'en admet que deux : plus et moins). La charge de "couleur» du quark (bleu, vert, rouge) correspond à un nombre quantique interne au quark.

Les objets graphiques de la physique quantique doivent donc être distingués des procédés de visualisation qui, à divers degrés, visent à exposer, représenter ou illustrer, une réalité physique externe ou expérimentale, comme c'est le cas, par exemple des coupes transversales, des images générées par ordinateur, des imageries médicales de type fMRI, ou des cartes géologiques. Je suis en désaccord sur ce point, avec E. RowleyJolivet lorsqu'elle reprend les propos de Martin Rudwick et présente les cartes géologiques comme des constructions théoriques ("highly theoretical constructs» M. Rudwick cité par E. Rowley-Jolivet $2002: 22$ ). Il s'agit selon moi au contraire d'un mode de projection d'une réalité géologique externe, couplé à une construction schématisée destinée à faciliter la lecture et l'analyse (choix d'échelle, et de couleurs), et non d'un mode de conceptualisation. Il importe donc de distinguer deux types d'objets images : d'une part les objets graphiques théoriques qui construisent une conceptualisation (comme c'est le cas en mathématique) et, d'autre part, des représentations imagées qui proposent à divers degrés une radiographie du réel (comme en médecine ou en biologie par exemple). En schématisant, il y aurait alors une distinction fondamentale entre le mode de représentation propre aux sciences expérimentales et les images présentes dans les sciences fortement mathématisées (comme la physique théorique et les mathématiques). L'écriture mathématique offre en effet l'exemple le plus immédiatement saisissable de formalisme graphique. Comme le rappelle René Thom, " on constate historiquement que les schémas mathématiques ont toujours préexisté aux exigences de l'expérience » $(1983: 31)$; ils ne peuvent donc en être une illustration 
ou une représentation. R. Thom ne donne qu'un seul contre-exemple celui de la "théorie ondulatoire de Fourier » et la transformation de Fourier qui lui est associée (1983 : 31). Les théories mathématiques font «monde " indépendamment de toute réalité physique observable. Et l'on conçoit alors aisément les objets graphiques mathématiques comme étant avant tout formels ${ }^{6}$.

Le statut des objets graphiques touche donc aussi à la différentiation entre disciplines théoriques et disciplines expérimentales. Pourtant la conceptualisation graphique n'est pas limitée aux sciences fortement mathématisées; elle est également présente dans des sciences dites expérimentales comme la chimie.

En chimie organique, une molécule comme l'éthanol peut être représentée dans une forme brute ( $\mathrm{C} 2 \mathrm{H} 6 \mathrm{O})$ qui est celle présentée dans les équations, ou par des méthodes de visualisation qui s'attachent soit à la représentation moléculaire (représentations de Cram), soit aux liaisons de valence (représentations de Lewis). Dans la représentation de Cram les « coins volants » (flying wedges), noirs ou hachurés, permettent d'évoquer la structure d'une molécule dans l'espace. Dans l'exemple présenté à la figure 2, les liaisons matérialisées par un trait fin sont dans le plan de la feuille, celles matérialisées par des coins noirs sont plus proches de l'observateur, alors que les liaisons hachurées sont en arrière-plan.

Figure 2. Représentation de Cram
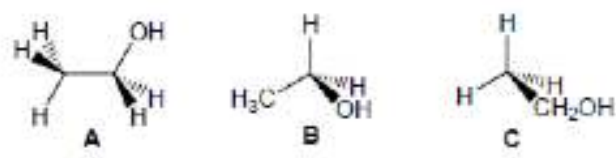

Les représentations de Lewis permettent de visualiser la " couche de valence ", et donc la structure électronique de l'atome qui est déterminante pour l'étude des réactions chimiques entre molécules (figure 3).

Figure 3. Représentations de Lewis

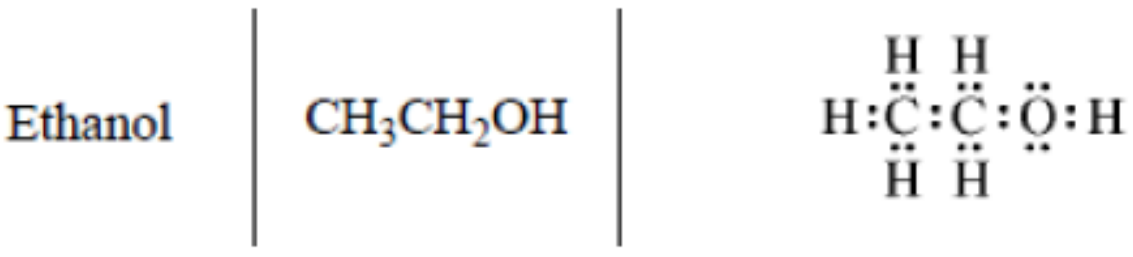

19 À première vue, les deux modes graphiques font référence à un réel externe: les orientations spatiales des molécules dans la représentation de Cram, et les couches électroniques externes des atomes (ou couches de valence) dans la représentation de Lewis. Pourtant, il s'agit moins de proposer une cartographie moléculaire (Cram) ou électronique (Lewis), que de poser un cadre accompagnant le type de développement recherché. Les représentations de Lewis définissent un ensemble de liaisons possibles, et délimitent la réactivité chimique des molécules. Les représentations de Cram singularisent les liaisons entre atomes, pour permettre d'étudier plus précisément l'évolution de l'architecture de la molécule lors de la réaction chimique. Le mode 
graphique utilisé est donc intimement lié au type de développement recherché. Il participe à la progression de la démonstration scientifique visée.

Aussi contre-intuitif que cela puisse paraître, les objets graphiques de la chimie organique s'apparentent à ceux de la physique théorique et des mathématiques; ils matérialisent les concepts sur la page et en sont le principe de construction. Ils se distinguent en cela des imageries médicales, qui visent avant tout à rendre visible ce qui est invisible à l'œil humain, sans chercher une quelconque élaboration théorique. Une radiographie existe indépendamment de la lecture qui peut en être faite par un médecin, indépendamment également des débats auxquels son interprétation peut donner naissance. Inversement, les images mathématiques concrétisent une construction théorique. Ils en sont une empreinte graphique au sens où Freud l'accorde au pas de la Gradiva de Jensen (Freud 1986). Derrida, relisant Freud, explique que dans la Gradiva, l'archéologue Hanold "veut exhumer une impression » qui « se confonde presque avec la pression du pas qui laisse sa marque encore vivante sur un support, une surface, un lieu d'origine ». Autrement dit, il rêve de «l'instant » où «la trace ne se distinguerait plus de son support » et cherche à retrouver l'instant, où «le pas vient faire encore un avec le subjectile» (1995: 150-152). La pensée scientifique vient aussi faire un avec le subjectile ${ }^{7}$. Selon la définition du Littré, le subjectile est la "surface externe sur laquelle le peintre applique une couche d'enduit, de peinture, de vernis. Pour la première couche, le subjectile s'identifie au matériau qui est appelé à recevoir le système de peintures. » Dans « Forcener le subjectile » Derrida envisage le subjectile comme un "lieu d'incubation", et c'est bien de cela qu'il s'agit aussi ici. Les objets images sont le lieu d'incubation de la pensée scientifique, ils en sont à la fois le support matériel et le lieu de sa construction.

21 Revenons à notre interrogation de départ : de quels outils disposent les humanités (au sens large) pour analyser ces objets graphiques? Il me semble que nos analyses des écrits scientifiques auraient à gagner à s'appuyer également sur les analyses philosophiques, plastiques et littéraires qui s'intéressent à ce qui fait image, forme et figure.

\section{Esquisse d'une nouvelle intégration disciplinaire}

Les réflexions proposées ici ne visent nullement l'exhaustivité, mais doivent être envisagées comme une première esquisse de pistes possibles. Au vu de ce qui précède, deux directions peuvent être évoquées : l'une a trait aux objets images conçus comme subjectile, et l'autre, plus immédiate sans doute et par laquelle je commencerai, tient à l'imbrication du visuel et du textuel en sciences.

Dans Picture Theory, W. J. T. Mitchell propose la notion d'imagetexte pour contester la dialectique « mot et image ». Au lieu de comparer deux modes d'écriture, d'envisager un tracé multimodal, ou même de dessiner un trait d'union entre image et texte qui relierait et décomposerait les deux gestes, l'imagetexte de W.J.T. Mitchell imagine un nouvel agencement, une forme composite et synthétique («a composite, synthetic form » 1994 : 83), qui à bien des égards est aussi celle de la science. C'est cette imagetexte scientifique qu'il nous reste à penser.

L'imagetexte nous incite à abandonner la lecture linéaire appelée par la plupart des textes, et à penser en termes de narratologie visuelle. Comme le souligne Jay Lemke, la sémiotique visuelle est non linéraire (« Spoken language is linear [...] but no visual 
semiotic can be » Lemke 2005 : 95). L'exemple dans la figure 4, tiré de "A method of recursive images to solve transient heat diffusion in multilayer materials » de C.J. Dias, permet une première saisie des différents modes de lecture que requiert l'imagetexte scientifique.

Figure 4. Imagetexte scientifique
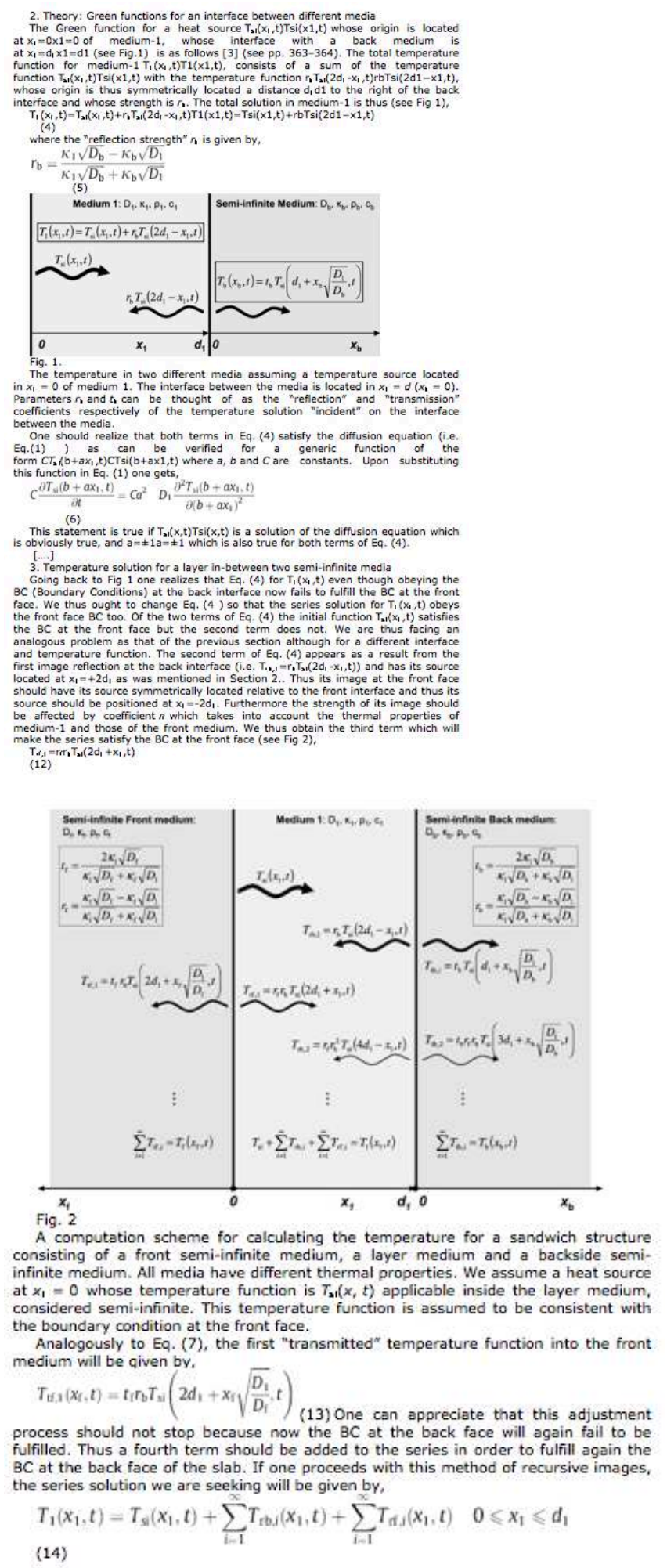

ensemble à parcourir de haut en bas et de droite à gauche. La «lecture " peut aussi commencer par celle du texte qui accompagne les figures et lui sert tout à la fois de titre, de description et d'explication. Du fait de son inscription sur l'espace de la feuille, l'image scientifique partage certaines caractéristiques de tout « objet d'art spatial », qui «ne prescrit pas nécessairement d'ordre de lecture» (Derrida 1978: 58). Toutefois, le « titre » d'une figure scientifique a un rôle distinct des titres des tableaux en art ou des sculptures. En peinture, comme le rappelle Liliane Louvel, « l'image peut jouer avec le titre, tenter de lui échapper. C'est le cas du leurre de La trahison des images de Magritte plus souvent connu sous le titre Ceci n'est pas une pipe. Faux titre qui depuis l'intérieur du tableau introduit un jeu de dupes ainsi qu'un jeu de mots un peu salace » $(2010: 68)$. Inversement, en science, le titre a un rôle programmatique et structurant : un graphe, sans légende et sans indications d'ordonnée et d'abscisse ne peut être interprété, et ne laisse même aucune possibilité de lui attribuer un sens. Même au sein du bloc graphique, l'image lue de manière isolée se dérobe et ne se laisse pas saisir. Pour autant, les légendes et les blocs textuels ne sont pas des cadres dans lesquels viendraient s'insérer des images. Il s'agit, à l'inverse, de penser ensemble les éléments textuels et graphiques que l'écriture scientifique déploie dans un même espace.

L'étude de W.J. T. Mitchell et la notion qu'il élabore permet, d'une part, de prendre en compte l'hétérogénéité des systèmes de représentation propres au visible et au lisible: " the heterogeneity of representational structures within the field of the visible and the readable " (1994: 88), mais elle permet aussi de dépasser cette hétérogénéité, pour envisager ensemble l'image et le texte. Si les exemples étudiés plus haut, les diagrammes de Feynman ou les représentations de Lewis et de Cram, peuvent se «lire " indépendamment de tout texte, ils sont avant tout une étape d'un développement théorique qui intègre texte et images.

S'intéresser aux traces écrites des écrits scientifiques nous incite à analyser le mouvement de la pensée, et le pouvoir de condensation des objets images, autrement dit la manière dont le sens s'y construit comme forme. La remarque de J. Derrida sur le subjectile (citée plus haut) attire notre attention sur le support papier ou écran, qui est le lieu du développement théorique. Pour Derrida, le subjectile est «un lieu d'incubation" (1986: 96) ou un «entre-deux». Le "subjectile n'a pas d'autre consistance que celle de l'entre-deux» (1986: 60), nous dit-il. La pensée se fonde au moment où elle se couche sur son support. Or, loin d'être un simple réceptacle, le 
subjectile tel que le théorise Derrida est un mouvement, une «jetée ». La force de " jetée » est

le mouvement qui, sans être jamais lui-même à l'origine, se modalise et se disperse dans les trajectoires de l'objectif, du subjectif, du projectile, de l'introjection, de l'interjection, de l'objection, de la déjection et de l'abjection, etc. Le subjectile se tient entre ces différentes jetées, soit qu'il en constitue l'élément sous-jacent, le lieu de naissance, soit qu'il s'interpose, comme une toile, un voile, un "support» de papier, l'hymen entre le dedans et le dehors, le dessus et le dessous, l'en-deçà ou l'au-delà, soit enfin qu'il devienne à son tour jetée, non pas cette fois comme le mouvement même de ce qui se jette mais comme la retombée durcie d'une masse de pierre inerte dans le port, la limite d'une "tempête arrêtée", le barrage. (Derrida $1986: 63$ )

Cette mise en mouvement de la pensée a également lieu dans les objets images théoriques scientifiques. Ce sont des formes qui portent en elles une force de mouvement.

La littérature et la philosophie se sont saisies de la matérialité du textuel et ont analysé un langage qui excède sa simple fonction de communication. Ces deux aspects qui semblent aux antipodes de la non-ambiguïté visée par la science peuvent pourtant nous être utiles. Le "pouvoir de figurer et pas seulement de signifier ", que Jean-François Lyotard note à propos du poème de Mallarmé Un coup de dés jamais n'abolira le hasard (2002: 62), est aussi celui des diagrammes de Feynman. Le « pouvoir de figurer » auquel J.-F. Lyotard fait référence est celui de la figure et du figural et non du figuratif : « la figure est une déformation qui impose à la disposition des unités linguistiques une autre forme » (2002: 61). La transposition aux sciences pourrait sembler d'autant plus paradoxale que la remarque de J.-F Lyotard est liée à Mallarmé, et à un écrit qui selon lui "dérobe radicalement le langage à sa fonction prosaïque de communication". Pourtant, J.-F Lyotard octroie au langage mallarméen « un pouvoir qui l'excède, le pouvoir d'être «vu» et pas seulement lu-entendu» (2002: 62), qui est aussi celui des formes scientifiques. Il rappelle, en outre, qu'il ne s'agit pas de faire comme si, les mots n'étaient pas des mots, mais qu'il faut prendre en considération la «signification de ces inscriptions bizarrement distribuées sur l'espace du volume » (2002:62).

Du discours, J.-F. Lyotard indique qu'il « ne signifie pas seulement " mais " exprime", « [e]t s'il exprime, c'est qu'il a lui aussi du bougé consigné en lui, du mouvement, de la force, pour soulever la table des significations par un séisme qui fait sens » (2002: 15). La pensée de J.-F. Lyotard sur les objets littéraires et la notion d'« énergétique » qui "plie » et «froisse le texte» $(2002: 14)$ peut à son tour être froissée et transposée aux objets scientifiques : la puissance de la ligne mathématique est son énergétique, elle est ce qui fait forme ou géométrie tout en étant une force d'abstraction. Elle est donc aussi "du bougé consigné ", et la saisie d'une énergétique. Les propos de Johanna Drucker sur le texte littéraire peuvent alors tout aussi bien caractériser le texte scientifique : « This does not make meaning. It only makes a space in which meaning comes to have its face pressed up against the glass, waiting to break through beyond the mirror.» (1998: 55)

Dans les entretiens qu'ils réalisent avec René Thom dans Paraboles et Catastrophes, Giulio Giorello et Simona Morini rappellent les raisons « administratives » et historiques de la séparation des disciplines, mais évoquent aussi une raison plus théorique. Convoquant Popper, ils soulignent que «les théories que nous élaborons pour résoudre nos problèmes tendent à s'accroître à l'intérieur de systèmes unifiés » (Thom 1983 : 19). Or, 
en structurant les champs de connaissance, nous les mettons aussi dans des boîtes étanches. J'ai souhaité faire travailler les cadres conceptuels habituels pour faire dialoguer l'anglais de spécialité et la critique littéraire ou artistique, et ouvrir ainsi une voie complémentaire de celle de l'analyse de discours.

\section{BIBLIOGRAPHIE}

Tous les liens hyperliens ont été consultés le 4 septembre 2015.

DERRIDA, Jacques. 1978. La Vérité en peinture. Paris : Flammarion.

DERRIDA, Jacques. 1986. « Forcener le subjectile ». Artaud. Dessins et portraits. Paris : Gallimard.

DERRIDA, Jacques. 1995. Mal d'archive : une impression freudienne. Paris : Galilée.

DIAS, C.J. 2015. «A method of recursive images to solve transient heat diffusion in multilayer materials ». International Journal of Heat and Mass Transfer 85, 1075-1083. [En ligne] <http:// www.sciencedirect.com/science/article/pii/S0017931015001568>.

DRUCKER, Johanna. 1998. Figuring the Word: Essays on Books, Writing, and Visual Poetics. New York : Granary Books.

FREUD, Sigmund. 1986. Le délire et les rêves dans la Gradiva de W. Jensen. Traduit par Paule Arbex \& Rose-Marie Zeitlin, Préface de J.-B. Pontalis. Paris : Gallimard.

LAFFONT, Hélène \& Jean-Louis Trouillon. 2013. « La recherche en anglais scientifique et la revue ASp : retour sur vingt ans de publications (1993-2012)». ASp 63, 113-158.

LEMKE, Jay. 2005 [1998]. « Multiplying meaning: visual and verbal semiotics in scientific texts ». In Martin, J.R. \& R. Veel (dir.), Reading Science: Critical and Functional Perspectives on Discourses of Science. New York: Routledge, 87-113.

LOUVEL, Liliane. 2010. Le Tiers pictural : Pour une critique intermédiale. Rennes : Presses Universitaires de Rennes.

LYOTARD, Jean-François. 2002 [1971]. Discours, figure. $5^{\mathrm{e}}$ tirage. Paris : Klincksieck.

MANDL, Franz \& Graham SHAW. 1984. Quantum Field Theory. New York : John Wiley \& Sons.

MANDELBROJT, Jacques. 2007. " La pensée gestuelle dans la science comme dans l'art ». Alliage 61, décembre, mis en ligne le 31 juillet 2012, <http://revel.unice.fr/alliage /index.html?id=3454>.

MÉMET, Monique et Michel VAN DER YEUGHT. 2008. «Bref historique de ASp, la revue du GERAS ». ASp «Les trente ans du GERAS », Numéro spécial, 25-28.

MITChelL, W. J. T. 1994. Picture Theory: Essays on Verbal and Visual Representation. Chicago : The University of Chicago Press.

MONOD, Jacques. 1970. Le Hasard et la nécessité. Paris : Seuil.

PETIT, Michel. 1997. «Stylistique(s) contrastive(s) du discours scientifique ». ASp 15-18, [En ligne] mis en ligne le 25 mai 2012, <http://asp.revues.org/3015>. 
ROSENBAUM, Alexis. 2009. «Sur le statut des diagrammes de Feynman en théorie quantique des champs », Philosophia Scientice 13-2 [En ligne], mis en ligne le 01 octobre 2009, <http:// philosophiascientiae.revues.org/301>.

ROWLEY-JOLIVET, Elizabeth. 2002. «Visual discourse in scientific conference papers. A genre-based study ». English for Specific Purposes 21/1, 19-40.

DE SAINT-OURS, Alexis. 2004. « Les sourires de l'être ». Penser par le diagramme. De Gilles Deleuze à Gilles Châtelet. TLE 22. Saint-Denis : Presses Universitaires de Vincennes, 29-53.

SABER, Anthony. 2014. « Éditorial ». ASp 66 [En ligne], mis en ligne le 06 octobre 2014, <http:// asp.revues.org/4521>.

SIONIS, Claude. 1997. «The integration of verbal and non-verbal materials in some instances of written scientific discourse ». ASp 15-18 [En ligne] mis en ligne le 18 novembre 2012, <http:// asp.revues.org/3191>.

THOM, René. 1983 [1980]. Paraboles et catastrophes. Paris : Flammarion.

\section{NOTES}

1. Le principe combinatoire qu'évoque Lemke provient de la pluralité des modes d'accès au sens dans les genres multimédia: «In multimedia genres, meanings made with each functional resource in each semiotic modality can modulate meanings of each kind in each other semiotic modality, thus multiplying the set of possible meanings that can be made (and so also the specificity of any particular meaning made against the background of this larger set of possibilities. » (Lemke 2005 : 92)

2. Cette approche se veut complémentaire des études qui optent pour un découpage disciplinaire.

3. Sur le même mode, Monique Mémet et Michel Van der Yeught entendent par analyses linguistiques « des articles qui traitent de l'analyse de la syntaxe, de l'analyse du discours, de stylistique, de phraséologie, d'analyse du genre, de lexicologie, de terminologie et de traduction » (2008: 27-28).

4. Forme est à entendre d'une part au sens graphique et pictural le plus courant et, d'autre part, au sens où l'emploie J.-F. Lyotard en lien avec les forces. «La force » pour J.-F. Lyotard «n'est jamais rien d'autre que l'énergie qui plie, qui froisse le texte et en fait une œuvre, une différence, c'est-à-dire une forme » (2002:14).

5. Richard Feynman a reçu le Prix Nobel en 1965 pour sa contribution fondatrice à l'électrodynamique quantique.

6. J'emploie ici formel au sens mathématique du terme.

7. Sur le subjectile chez Derrida, on pourra se reporter à « Forcener le subjectile ». Artaud. Dessins et Portraits. (Paris : Gallimard, 1986).

\section{RÉSUMÉS}

Analyser les écrits scientifiques nécessite de s'attacher à la manière dont les formes visuelles et textuelles s'entrelacent. Après un retour sur les études existantes, l'article envisage les limites 
des approches linguistiques centrées sur les composantes verbales et textuelles du discours scientifique. À l'inverse, cette étude se propose de se pencher sur le faire image scientifique, au travers notamment de l'exemple des diagrammes de Feynman. Il s'agira ainsi d'analyser la manière dont le sens se construit comme forme. La dernière partie de l'article se propose de reposer différemment le problème des outils théoriques dont disposent les humanités pour analyser les écrits scientifiques. Elle suggère d'intégrer les outils plus littéraires ou philosophiques que sont ceux de l'analyse de l'image (Louvel, Derrida, Lyotard) ainsi que de transposer et d'étendre aux sciences l'imagetexte de W.J.T. Mitchell.

In order to analyze scientific writing, one needs to focus on the way the visual and textual elements are interwoven. After considering previous studies, this article ponders upon the limits of linguistic approaches that concentrate on the verbal-textual aspects of scientific discourse. By taking the example of Feynman graphs, this study then seeks to explore how science images meaning, and how meaning is constructed as form (rather than text). The last part of this study examines the theoretical tools at our disposal to analyze scientific writing. It suggests opening up to the literary and philosophical tools of image analysis (Louvel, Derrida, Lyotard), and offers to build on W.J.T. Mitchell's imagetext so as to transpose it to the scientific field.

\section{INDEX}

Mots-clés : diagramme de Feynman, écriture scientifique, faire image, figure, imagetexte, trace Keywords : Feynman graph, figuring, imagetext, imaging, scientific writing, trace

\section{AUTEUR \\ GWEN LE COR}

Gwen Le Cor est maître de conférences HDR à l'université Paris 8. Membre de l'EA 1569 «Transferts critiques et dynamique des savoirs », elle est porteur du projet triennal « Text/ures : l'objet livre du papier au numérique », projet soutenu par le Labex Arts-H2H. Ses recherches récentes portent l'entre-deux de la littérature et des sciences, sur l'anglais de spécialité scientifique ainsi que sur la littérature américaine contemporaine. Outre une monographie (à paraître) sur l'œuvre de Jonathan Safran Foer, elle a publié plusieurs articles sur les œuvres numériques et imprimées de Jen Bervin, Percival Everett, Jonathan Safran Foer, Flannery O'Connor, Art Spiegelman, Stephanie Strickland, Steve Tomasula et Robert Penn Warren. Elle a également travaillé sur l'intersémioticité, le discours scientifique, les intersections littératuremathématiques, l'interface littérature-informatique, et plus largement le domaine des humanités numériques.gwen.le-cor@univ-paris8.fr 